\title{
Hip Fractures in Malta: Are we Missing an Opportunity?
}

\author{
Mark Bugeja, MD, MRCSEd ${ }^{1}$ Arthur Curmi, MD ${ }^{1}$ Daniel Desira, MD ${ }^{1}$ \\ Gregory Apap Bologna, MD, MRCSEd ${ }^{1}$ Francesco Galea, MD ${ }^{1} \quad$ Ivan Esposito, MD, FRCS, DSMRCS, FFSEM ${ }^{1}$ \\ ${ }^{1}$ Department of Trauma and Orthopaedics, Mater Dei Hospital, Malta \\ Address for correspondence Arthur Curmi, MD, Mater Dei Hospital, \\ Surg J (NY) 2021;7:e184-e190. \\ Msida MSD 2090, Malta (e-mail: arthurcurmi@gmail.com).
}

\begin{abstract}
\section{Keywords}

- hip fractures

- fragility fractures

- calcium

- vitamin D

- bisphosphonates

Introduction Osteoporosis is a bone disease that is both preventable and treatable. It usually becomes evident when a fragility fracture occurs. Unfortunately, most studies show that only a small percentage of individuals at increased risk of fracture are assessed and treated, even following a fragility fracture.

Objective The aim of this study was to determine whether patients suffering from a low-energy hip fractures in the Maltese Islands are given osteoporosis treatment.

Method All patients older than 50 years presenting to the acute care hospitals in Malta and Gozo with a fragility hip fracture during December 1, 2015 and November 30, 2016 were included. Data on mortality, other fragility fractures, prescription of calcium, vitamin D, and antiresorptive therapy were collected.

Results Calcium with vitamin D supplements were prescribed to $40 \%$ of patients; however, only $2.64 \%$ of patients were given pharmacological therapy. Following a hip fracture, the mortality rate was $18.5 \%$ at 1 year and $26.21 \%$ at 2 years. Apart from a high mortality rate, $28.19 \%$ of individuals sustained another fragility fracture before or after the hip fracture.

Conclusion There should be increased osteoporosis awareness in Malta and a national bone mineral density screening program should be set up. An active role of the orthogeriatrics team in the management and treatment of osteoporosis following a fragility fracture might improve treatment rate and decrease refracture and mortality rates.
\end{abstract}

At the consensus development conference in 1990, osteoporosis was defined as " $a$ disease characterized by low bone mass, microarchitectural deterioration of bone tissue leading to enhanced bone fragility, and a consequent increase in fracture risk." It is referred to as a "silent disease," as it is usually asymptomatic, and becomes evident when a fragility fracture occurs. ${ }^{2-5}$ Hence, the clinical importance of this condition rests on the fragility fractures that arise. ${ }^{6}$ These fragility fractures occur in the lumbosacral spine, distal radius, hip, and proximal humerus. Since bone mineral density (BMD) is low, the risk of fractures at other sites is

received

January 24, 2020

accepted after revision

April 14, 2021 also increased. ${ }^{6}$ Of these, hip fractures are the most serious, causing great social and economic burdens, and leading to increased morbidity, mortality, and disability, with most of these patients becoming institutionalized..$^{7-12}$ Melton ${ }^{13}$ estimated the lifetime risk of hip fracture at 17.5 and $6 \%$ in 50 year-old white women and men, respectively ( - Table 1 ). The risk is comparable to the other major fragility fractures involving the spine and wrist. ${ }^{13}$ Following an osteoporotic fracture, one is at an increased risk for further fragility fractures. ${ }^{14,15}$ To prevent this, several guidelines for the diagnosis and treatment of osteoporosis were developed.

\section{(C) 2021. The Author(s)}

This is an open access article published by Thieme under the terms of the Creative Commons Attribution License, permitting unrestricted use, distribution, and reproduction so long as the original work is properly cited. (https://creativecommons.org/licenses/by/4.0/)

Thieme Medical Publishers, Inc., 333 Seventh Avenue, 18th Floor, New York, NY 10001, USA 
Table 1 Estimated lifetime fracture risk in 50-year-old white women and men

\begin{tabular}{|l|l|l|}
\hline & $\begin{array}{l}\text { Women \% } \\
(95 \% \mathrm{Cl})\end{array}$ & $\begin{array}{l}\text { Men \% } \\
(95 \% \mathrm{CI})\end{array}$ \\
\hline $\begin{array}{l}\text { Proximal femur } \\
\text { fracture }\end{array}$ & $17.5(16.8,18.2)$ & $6.0(5.6,6.5)$ \\
\hline Vertebral fracture & $15.614 .8,16.3)$ & $5.0(4.6,5.4)$ \\
\hline $\begin{array}{l}\text { Distal forearm } \\
\text { fracture }\end{array}$ & $16.0(15.2,16.7)$ & $2.5(2.2,3.1)$ \\
\hline Any of the three & $39.7(38.7,40.6)$ & $13.1(12.4,13.7)$ \\
\hline
\end{tabular}

Abbreviation: $\mathrm{Cl}$, confidence interval.

Source: Table taken from Melton. ${ }^{13}$

However, its management remains a challenge, especially in the management of secondary causes, effectiveness and treatment period of antiresorptive agents, and the role of calcium and vitamin D supplements. ${ }^{16}$

\section{Detection of Disease}

In 1994, the World Health Organization (WHO) study group described osteoporosis in white postmenopausal women as a BMD value of 2.5 standard deviations (SD) or more below the young adult mean (T-score $\leq-2.5$ ). In the presence of one or more fragility fractures and a BMD value more than 2.5 SD below the young adult mean, it is defined as severe osteoporosis. ${ }^{17}$ For these diagnostic categories, BMD is converted into a T-score, which indicates the number of SD above or below the mean in healthy young adults ( - Table 2 ).$^{18}$ A major problem with BMD measurement is that, alone it is inadequate for the detection of individuals at high risk of fracture, with tests having high specificity but low sensitivity. ${ }^{19}$ Undeniably, most fragility fractures occur with a negative test. ${ }^{19}$

$\mathrm{X}$-ray absorptiometry is the most commonly used technique for measuring BMD. This utilizes the high sensitivity of calcium in absorbing X-rays to measure the relative amount in bones and soft tissue, hence, calculating bone mineral content and density. ${ }^{20} \mathrm{BMD}$ at the hip is the most accurate for predicting risk of hip fractures, with spinal BMD used for monitoring treatment. This is because measurements at the lumbar spine can be heavily influenced by artifacts. $^{20}$ In cases where the hip or spine cannot be measured or inter-

Table 2 WHO four general diagnostic categories in osteoporosis $^{17,18}$

\begin{tabular}{|l|l|}
\hline Diagnostic categories & BMD T-score \\
\hline Normal & -1.0 or above \\
\hline Low bone mass (osteopenia) & Between -1.0 and -2.5 \\
\hline Osteoporosis & -2.5 or below \\
\hline $\begin{array}{l}\text { Severe osteoporosis } \\
\text { (established osteoporosis) }\end{array}$ & $\begin{array}{l}\text { Below }-2.5 \text { in the } \\
\text { presence of one or } \\
\text { more fragility fractures }\end{array}$ \\
\hline
\end{tabular}

Abbreviations: BMD, bone mineral density; WHO, World Health Organization. preted, the forearm is used instead; however, there can be significant differences in BMD between the dominant and nondominant arm. ${ }^{20}$

\section{Epidemiological Risk Factors}

There are other risk factors other than BMD that predispose to fragility fractures. These include age, sex, diseases, and medications affecting bone strength, prior fragility fracture, family history of fragility fracture, body mass index, history of falls, smoking, alcohol, and physical inactivity. ${ }^{16,19}$ Peak bone mass occurs by the age of 30 but this decreases steadily after 40 years of age and continues at an accelerated rate around the menopausal years before slowing down again after the age $70 .{ }^{21}$ Age is therefore the strongest predictor for fracture risk. The FRAX (www.shef.ac.uk/FRAX/) and QFracture (www.qfracture.org) are two major scores based on risk factors and used to estimate the 10 -year probability of fracture. ${ }^{16}$

\section{Guidelines}

The American Association of Clinical Endocrinologists and American College of Endocrinology in 2016 published clinical practice guidelines on the diagnosis and treatment of postmenopausal osteoporosis (-Table 3). ${ }^{22}$ One of the criteria stated, that in the presence of a low energy spine or hip fracture, regardless of BMD, patients are at a high risk for further fractures, thus, should be diagnosed with osteoporosis and considered for treatment. ${ }^{22}$ The European Society of Endocrinology recommends pharmacological treatment to be started without a delay in patients with recent fractures to prevent further fragility fractures. ${ }^{23}$ Both the National Osteoporosis Foundation (NOF) and the UK National Osteoporosis Guideline Group (NOFF) recommend starting treatment following a fragility fracture. ${ }^{4,24}$

\section{Treatment Options}

As an adjunct to the osteoporosis therapies mentioned below, patients should be counseled on adequate calcium

Table 32016 AACE/ACE diagnostic criteria for osteoporosis in postmenopausal women

\begin{tabular}{|l|l|}
\hline 1 & $\begin{array}{l}\text { T-score }-2.5 \text { or below in the lumbar spine, } \\
\text { femoral neck, total, and/or 33\% radius }\end{array}$ \\
\hline 2 & $\begin{array}{l}\text { Low-trauma spine or hip fracture } \\
\text { (regardless of BMD) }\end{array}$ \\
\hline 3 & $\begin{array}{l}\text { Osteopenia or low bone mass } \\
\text { (T-score between }-1 \text { and }-2.5) \text { with a fragility } \\
\text { fracture of proximal humerus, pelvis, or possibly } \\
\text { distal forearm }\end{array}$ \\
\hline 4 & $\begin{array}{l}\text { Low bone mass or osteopenia and high FRAX } \\
\text { fracture probability based on country-specific } \\
\text { thresholds }\end{array}$ \\
\hline
\end{tabular}

Abbreviations: AACE/ACE, American Association of Clinical Endocrinologists/American College of Endocrinology; BMD, bone mineral density.

Source: Taken from Camacho et al. ${ }^{22}$ 
and vitamin D intake, fall prevention strategies, stopping smoking, limiting alcohol intake, and regular physical activity. $^{22,23}$

\section{Calcium}

Lifelong sufficient intake of calcium is needed to achieve peak bone mass and later maintenance of bone health. When intake is inadequate, in an attempt to maintain serum calcium, the body resorbs bone, decreasing the BMD. According to NOF men between the age of 50 and 70 years should consume $1000 \mathrm{mg}$ of calcium daily, while women older than 50 and men older than 70 should consume $1200 \mathrm{mg}$ of calcium daily. ${ }^{4}$ The most commonly used calcium supplements are calcium citrate and calcium carbonate, both of which are equally well absorbed when taken with food. ${ }^{25}$

\section{Vitamin D}

Vitamin D has a key role in calcium homeostasis. According to the NOF, the daily intake of vitamin D for those older than 49 years is between 800 and 1,000 international units (IU). ${ }^{4}$ Unfortunately, $90 \%$ of those aged between 51 and 79 years have inadequate intake of vitamin D. ${ }^{25}$ In view of variations in vitamin $D$ requirements between individuals, the dose of vitamin D supplements should be titrated according to serum 25(OH)D levels. In fact, many osteoporotic patients require more than what the NOF recommends. ${ }^{4}$ Vitamin D deficiency may be treated with $7,000 \mathrm{IU}$ vitamin $\mathrm{D}_{2}$ or vitamin $D_{3}$ for 8 to 12 weeks to attain a serum $25(\mathrm{OH}) \mathrm{D}$ level of around $30 \mathrm{ng} / \mathrm{mL}^{4}$ This is followed by maintenance dose (usually 1,500-2,000 IU daily) adjusted according to target serum 23(OH)D level. ${ }^{4}$

\section{Bisphosphonates}

Bisphosphonates are stable analogues of pyrophosphate with powerful effects on bone metabolism. ${ }^{26}$ They preferentially bind to the surface of bone at locations of active remodeling. In the acidic environment of the bone resorption sites, bisphosphonates detach form the bone mineral, attach to osteoclasts, and impair their function. ${ }^{26}$ Bisphosphonates may cause renal impairment and very rarely may cause osteonecrosis of the jaw. ${ }^{4}$ Long-term use of bisphosphonates is associated with low-energy atypical femur fractures. ${ }^{4}$ Thus, fracture risk should be recalculated after 3 to 5 years, with women who remain at high risk of fractures continuing treatment, while those having low-to-moderate risk of fractures being considered for a "bisphosphonate holiday" (temporary cessation of bisphosphonate for up to 5 years). ${ }^{23}$

\section{Denosumab}

Denosumab is a human monoclonal antibody against the receptor activator of nuclear factor kappa- $\beta$ ligand, thus, interfering with the formation, activation, and survival of osteoclasts. ${ }^{5,24,27}$ Denosumab is given subcutaneously every
6 months. Patients on denosumab can develop hypocalcemia; thus, sufficient calcium and vitamin D intake is of paramount importance, especially in those with severe kidney impairment. ${ }^{24}$ Also, serum calcium levels should be checked before every dose of denosumab and within 2 weeks after initial dose. ${ }^{24}$ Similar to bisphosphonates, denosumab can also cause osteonecrosis of the jaw and atypical femur fractures. ${ }^{24}$ Patients on denosumab should have fracture risk recalculated after 5 to 10 years, and women who remain at high risk of fractures either continue or else change therapy. ${ }^{23}$

\section{Teriparatide and Abaloparatide}

Teriparatide is parathyroid hormone $(\mathrm{PTH})$, while abaloparatide is a PTH-related protein analog. ${ }^{23}$ These are the two licensed peptides that are anabolic for bone (i.e., increase bone formation) when administered intermittently. ${ }^{23}$ Both agents may cause an increase in serum calcium levels; thus, serum calcium level should be checked before starting treatment. ${ }^{23}$ The European Society of Endocrinology recommends treatment with these agents for 2 years, then continue with antiresorptive medication to maintain the BMD gains. $^{23}$

\section{Selective Estrogen Receptor Modulators}

Selective estrogen receptor modulators (e.g., raloxifene) are agents that interact with intracellular estrogen receptors in target organs as estrogen agonists and antagonists. ${ }^{28}$ When compared with menopausal hormone therapy, it has less impact on BMD. ${ }^{23}$ They are indicated for postmenopausal women with osteoporosis in whom bisphosphonates or denosumab are not appropriate and who have low risk of thrombosis and high risk of breast cancer. ${ }^{22,23}$

\section{Menopausal Hormone Therapy}

According to the European Society of Endocrinology, menopausal hormone therapy should only be considered in women younger than 60 years or who are less than 10 years following menopause in whom bisphosphonates or denosumab are not indicated. ${ }^{23}$ Patients should not have any history of myocardial infarction, cerebrovascular accidents, breast cancer, or risk of thrombosis. ${ }^{23}$ Estrogen on its own should only be used in women with hysterectomy. ${ }^{22,23}$

\section{Calcitonin}

Calcitonin should only be used in patients in whom the above-mentioned therapies are not appropriate.,22,23 It is administered either as nasal spray (200 IU daily) or injected subcutaneously or intramuscularly (100 IU daily). Skin testing is recommended before using calcitonin to avoid hypersensitivity. ${ }^{22}$ In view of concerns about increased risk of cancer especially liver and prostate, the European Medicines Agency and Health Canada have withdrawn the nasal spray from the market. ${ }^{23}$ 
Table 4 Calcium, vitamin D supplements, and antiresorptive medications

\begin{tabular}{|l|l|l|l|l|}
\hline & Women $(\boldsymbol{n}=\mathbf{3 3 0})$ & Men $(\boldsymbol{n}=\mathbf{1 2 4})$ & Total & Percentage \\
\hline Calcium and vitamin D & 143 & 39 & 182 & 40 \\
\hline Calcium only & 15 & 8 & 23 & 5 \\
\hline No supplements & 172 & 77 & 249 & 55 \\
\hline Antiresorptive medications & 10 & 2 & 12 & 2.64 \\
\hline
\end{tabular}

\section{Objective of the Study}

Hip fractures in Malta are managed using a multidisciplinary team; unfortunately, orthopaedic surgeons often rely on primary care physicians to take responsibility for the management of osteoporosis. ${ }^{29}$ Since these fractures are usually admitted, orthopaedic surgeons are the first contact for these patients, and this gives them the opportunity not only to treat the fracture but also to start osteoporosis treatment. ${ }^{3,14}$ The objective of this observational study is to determine whether patients sustaining low-energy hip fracture in the Maltese Islands were given osteoporosis treatment.

\section{Method}

All individuals older than 50 years presenting to the acute care hospitals in Malta and Gozo with low-trauma hip fractures during December 1, 2015 and November 30, 2016 were included in this study. They were identified retrospectively using the hospitals' imaging software, Picture Archiving and Communication System. Using the same software, prior (10 years before) and subsequent fragility fractures of the hip, distal radius, proximal humerus, and spine were recorded. Using the hospitals' electronic case summary software, the list of medications on discharge from both the acute hospitals and the rehabilitation hospital was obtained. From the drug history on the discharge summary, those prescribed calcium, vitamin $\mathrm{D}$, and antiresorptive therapy were noted. Patients' demographics, date deceased, and length of hospital stay were obtained using the hospitals' i. Clinical Manager (i.CM) version 2.1. The in-hospital, 1-year, and 2-year mortality following the hip fracture was calculated.

\section{Results}

There were 454 individuals older than 50 years who suffered from a low-energy hip fracture between December 1, 2015 and November 30,2016 . The average age at presentation was 80.3 years with two-thirds $(72.7 \% ; n=330)$ being women. Calcium and vitamin D supplements were given to $40 \%$ of individuals $(n=182)$. Calcium supplements on their own were given to $5 \%$ of patients $(n=23)$. The majority $(55 \%$; $n=249$ ) were not given any supplements ( - Table 4 ).

Only 12 patients (2.64\%) were given osteoporosis therapy (-Table 4). Most of these patients $(n=9)$ were prescribed therapy from a rehabilitation hospital, two patients were already on treatment when they suffered from a hip fracture, while only one patient was prescribed treatment form the
Table 5 Fragility fractures prior or subsequent to the hip fracture

\begin{tabular}{|l|l|}
\hline $\begin{array}{l}\text { Prior or subsequent fragility } \\
\text { fracture }\end{array}$ & $\begin{array}{l}\text { No. of } \\
\text { individuals (\%) }\end{array}$ \\
\hline Contralateral hip fracture & $39(8.59)$ \\
\hline Fracture of the distal radius & $39(8.59)$ \\
\hline Proximal humerus fracture & $37(8.15)$ \\
\hline Spinal fracture & $13(2.86)$ \\
\hline Total & $128(28.19)$ \\
\hline
\end{tabular}

acute hospital. Of the osteoporosis treatments, bisphosphonates were the most prescribed, and only one patient received the monoclonal antibody denosumab. The bisphosphonate of choice was alendronic acid. This was given to eight patients. The other three patients receiving bisphosphonates were given zoledronic acid, risedronate sodium, and ibandronic acid.

The in-hospital mortality was 5.95\% $(n=27)$, which rose to $18.50 \%(n=84)$ by 1 year, and $26.21 \%(n=119)$ after 2 years following the hip fracture. Apart from having an increased risk of mortality, more than one in four patients $(n=128 ; 28.19 \%)$ suffered from a fragility fracture before or subsequent to the hip fracture (-Table 5). Thirty-nine patients (8.59\%) suffered from another hip fracture, while the same number of patients $(n=39)$ suffered from a fracture of the distal radius. Thirty-seven patients (8.15\%) suffered from a proximal humerus fracture, while spine fractures were present in only 13 patients $(2.86 \%)$.

Most patients suffering from hip fractures are elderly with multiple comobilities taking several medications. Polypharmacy is considered a crucial risk factor for falls in older people, with the risk of falls increasing with the number of medicines taken per day. ${ }^{30}$ Certain medicines are associated with increased risk of falls. These include antihypertensives, diuretics, antipsychotics, and antidepressants. ${ }^{30}$ On the other hand, there are medications that decrease the BMD. These include proton pump inhibitors, glucocorticoids, anticoagulants, insulin, and oral hypoglycemic agents. ${ }^{31}$-Table 6 shows the frequency of these commonly prescribed medicines.

\section{Discussion}

The aim of pharmacological therapy in the treatment of osteoporosis is to prevent fractures. The Fracture Intervention Trial (FIT) Research Group in a randomized, doubleblind, placebo-controlled trial consisting of 2,027 women 
Table 6 Commonly prescribed drugs that may increase the risk of falls or decrease bone mineral density

\begin{tabular}{|l|l|}
\hline Type of drug & $\begin{array}{l}\text { No. of individuals taking } \\
\text { these medications (\%) }\end{array}$ \\
\hline Antihypertensives & $247(54.41)$ \\
\hline Diuretics & $174(38.33)$ \\
\hline Antidepressants & $73(16.08)$ \\
\hline Antipsychotics & $51(11.23)$ \\
\hline Proton pump inhibitors & $157(34.58)$ \\
\hline Glucocorticoids & $20(4.41)$ \\
\hline Anticoagulants & $108(23.79)$ \\
\hline Antiplatelets & $126(27.75)$ \\
\hline Oral hypoglycemic agents & $103(22.69)$ \\
\hline Insulin & $42(9.25)$ \\
\hline
\end{tabular}

with at least one vertebral fracture tested the hypothesis that alendronate decreases the risk of new vertebral fracture during a 3-year follow-up. ${ }^{32}$ The risk of suffering from a further vertebral fracture was $8 \%$ in the alendronate group and $15 \%$ in the placebo group. The risk of other fracture was also lower in the alendronate group (13.6 vs. $18.2 \%){ }^{32}$ Another study comprising of 4,432 postmenopausal women from the FIT tested the hypothesis that taking alendronate for 4 years would decrease the risk of vertebral fractures in women who have low BMD but no vertebral fractures. ${ }^{33}$ There was a $36 \%$ reduction in vertebral fractures in the alendronate group, and it was reported that alendronic acid increased BMD at all sites as measured by dual X-ray absorptiometry. ${ }^{33}$ In another controlled, double-blind, multicenter study from FIT found that alendronate is effective in lowering the risk of vertebral fractures in women with $\mathrm{T}$ scores of -1.6 to -2.5 (i.e., do not meet the BMD criterion for osteoporosis). ${ }^{34}$ This study consisted of 3,737 postmenopausal women, with the risk of vertebral fracture being significant lower in the alendronate group

Despite good evidence that pharmacological therapy for osteoporosis reduces the risk of fragility fractures, unfortunately, several studies reported a low rate of initiation of osteoporotic treatment following a hip fracture. Jennings et $\mathrm{al}^{35}$ conducted a study comprising of 51,346 patients older than 64 years from 318 hospitals in the United States suffering from a fragility fracture of the hip between October 2003 and September 2005. Calcium and vitamin D were prescribed to $6.6 \%$ ( $n=3,405)$ of the patients, while antiresorptive or bone-forming drugs were given to $7.3 \%(n=3,763)$ of patients. However, only $2 \%(n=1,023)$ received the ideal treatment (i.e., calcium and vitamin $\mathrm{D}$ together with an antiresorptive or bone-forming drug). Gonnelli et al $^{12}$ performed a retrospective study on 697 patients from four centers in Italy (Siena, Verona, Naples, and Palermo) with a low-energy hip fracture during the year 2014. Calcium and vitamin D were started in $18.8 \%$ of patients, while only $9.7 \%$ of patients were started on antiresorptive or bone-forming medications.
Cuschieri et $\mathrm{al}^{36}$ conducted a retrospective study on all patients over the age of 60 years who suffered from a lowenergy hip fracture in Malta in the year 2011. None of the patients were prescribed calcium, vitamin D, or bisphosphonates during their stay in the acute hospital. Further fractures of the hip within a year occurred in $6 \%$ of the individuals, with the authors highlighting the financial burden of these refractures. These results are similar to our study where only one patient was started on treatment during the stay in the acute hospital, and on discharge from rehabilitation hospital, only $2.6 \%$ of patients were prescribed osteoporosis medications.

For osteoporosis treatment to work, patients need to be compliant with treatment. Huybrechts et $\mathrm{al}^{37}$ in their study found that three-quarters of the patients had medium to poor compliance with osteoporosis treatment. Low compliance was associated with a $37 \%$ higher risk of hospitalization and a $17 \%$ increase in fracture rate. Rabenda et $\mathrm{al}^{38}$ conducted a study on all postmenopausal women suffering from a hip fracture between April 2001 and June 2001 in the entire Belgian population. This consisted of 23,146 individuals. Only $6 \%$ received pharmacologic osteoporosis treatment (4.6\% given alendronate, $0.7 \%$ given risedronate, and $0.7 \%$ given raloxifene). At 6 months, 40\% stopped taking alendronate, while at 1 year only $41 \%$ of those prescribed alendronate were compliant.

Once-yearly regimen with intravenous bisphosphonates that do not require individuals to remain standing and are easier to tolerate might improve compliance. Lyles et $\mathrm{al}^{39}$ in a randomized, double-blind, placebo-controlled trial found that a yearly zoledronic acid infusion within 3 months following a hip fracture was associated with lower risk of new fractures and decreased mortality. In this trial, 1,065 individuals received $5 \mathrm{mg}$ intravenous zoledronic acid yearly, while 1,062 individuals received a placebo. The rates of new fragility fractures were $13.9 \%$ in the placebo group and $8.6 \%$ in the zoledronic acid group (i.e., a 5.3\% absolute risk reduction and a $35 \%$ relative reduction). Mortality rate in the placebo group was $13.1 \%$, while in the zoledronic acid group it was $9.6 \%$ (i.e., $28 \%$ reduction in mortality). There were no reported jaw osteonecrosis or problems with fracture healing in this trial.

The findings of our study must be seen in light of some limitations. Data was collected retrospectively, assuming the drug history on the discharge letter was correct. We suggest the issuing of local guidelines on the prescription of antiosteoporosis medication following fragility fractures. Following this, prospective audits should be performed ensuring adherence to the guidelines and patients' compliance to medications. Unfortunately, despite the Maltese Islands having a free national healthcare system, antiosteoporosis medications are not on the free medication entitlement scheme; thus, anti-osteoporosis medications should be prescribed routinely following low-energy hip fractures, and this will negatively affect compliance.

\section{Conclusion}

Osteoporosis is a bone disease that is both preventable and treatable. Unfortunately, most studies worldwide show that 
only a small percentage of those at increased risk of fracture are assessed and treated, even following a fragility fracture. The rate of osteoporosis treatment in Malta is no different with only $2.64 \%$ of the studied population receiving osteoporosis therapy. Knowing that fragility fractures are of a great social and financial burden with a high morbidity and mortality rate, it is important that this issue be addressed. An active role of the orthogeriatrics team in the management and treatment of osteoporosis might improve treatment rate and decrease refracture and mortality rates. There should be increased osteoporosis awareness in Malta and a national BMD screening program should be set up.

\section{Declaration of Conflicting Interests}

The authors declared no potential conflicts of interest with respect to the research, authorship, and/or publication of this article.

\section{Funding}

The authors received no financial support for the research, authorship, and/or publication of this article.

\section{References}

1 Consensus development conference. Consensus development conference: prophylaxis and treatment of osteoporosis. Am J Med 1991;90(01):107-110

2 Sözen T, Özışık L, Başaran NÇ An overview and management of osteoporosis. Eur J Rheumatol 2017;4(01):46-56

3 Larsson S, Fazzalari NL. Anti-osteoporosis therapy and fracture healing. Arch Orthop Trauma Surg 2014;134(02):291-297

4 Cosman F, de Beur SJ, LeBoff MS, et al; National Osteoporosis Foundation. Clinician's guide to prevention and treatment of osteoporosis. Osteoporos Int 2014;25(10):2359-2381

5 Kling JM, Clarke BL, Sandhu NP. Osteoporosis prevention, screening, and treatment: a review. J Womens Health (Larchmt) 2014;23 (07):563-572

6 Kanis JA, Melton LJ III, Christiansen C, Johnston CC, Khaltaev N. The diagnosis of osteoporosis. J Bone Miner Res 1994;9(08): 1137-1141

7 Bugeja M, Aquilina S, Farrugia C, Esposito I. Demographic study of hip fractures in the Maltese Islands. Geriatr Orthop Surg Rehabil 2018;9:2151459318764772

8 Akkawi I, Zmerly H. Osteoporosis: current concepts. Joints 2018;6 (02):122-127

9 Pietri M, Lucarini S. The orthopaedic treatment of fragility fractures. Clin Cases Miner Bone Metab 2007;4(02):108-116http://www. ncbi.nlm.nih.gov/pubmed/22461210. Accessed May 4, 2021

10 Porter JL, Varacallo M. Osteoporosis. StatPearls Publishing2019 http://www.ncbi.nlm.nih.gov/pubmed/28722930.Accessed May 4, 2021

11 Kim S-R, Ha Y-C, Park Y-G, Lee S-R, Koo K-H. Orthopedic surgeon's awareness can improve osteoporosis treatment following hip fracture: a prospective cohort study. J Korean Med Sci 2011;26 (11):1501-1507

12 Gonnelli S, Caffarelli C, Iolascon G, et al. Prescription of antiosteoporosis medications after hospitalization for hip fracture: a multicentre Italian survey. Aging Clin Exp Res 2017;29(05): 1031-1037

13 Melton LJ, Chrischilles EA, Cooper C, Lane AW, Riggs BL. Perspective how many women have osteoporosis? J Bone Miner Res 1992; 07(09):1005-1010

14 Elliot-Gibson V, Bogoch ER, Jamal SA, Beaton DE. Practice patterns in the diagnosis and treatment of osteoporosis after a fragility fracture: a systematic review. Osteoporos Int 2004;15(10): 767-778

15 Kim SC, Kim MS, Sanfélix-Gimeno G, et al. Use of osteoporosis medications after hospitalization for hip fracture: a cross-national study. Am J Med 2015;128(05):519-26.e1

16 Dilemmas in the management of osteoporosis. Drug Ther Bull 2015;53(02):18-21

17 World Health Organization. (1994). Assessment of fracture risk and its application to screening for postmenopausal osteoporosis: report of a WHO study group [meeting held in Rome from 22 to 25 June 1992]. World Health Organization https://apps.who.int/iris/ handle/10665/39142. Accessed December 10, 2019

18 Szulc P, Bouxsein ML. Overview of osteoporosis: epidemiology and clinical management. Vertebral fracture initiative resource document. 2011. Accessed December 10, 2019 from: www.iofbonehealth.org/IOF_VFI-Part_I

19 WHO Scientific Group on The Assessment of Osteoporosis at Primary Health Care Level Summary Meeting Report.; 2004. Accessed December 10, 2019 from: https://www.who.int/chp/ topics/Osteoporosis.pdf

20 Sheu A, Diamond T. Bone mineral density: testing for osteoporosis. Aust Prescr 2016;39(02):35-39

21 Diamond T, Tonks K. Secondary causes of osteoporosis in women: diagnoses not to be missed. Med Today (Karachi) 2008;9(05): 48-62

22 Camacho PM, Petak SM, Binkley N, et al. AACE/ACE Guidelines American Association of Clinical Endocrinologists and American College of Endocrinology Clinical Practice Guidelines for The Diagnosis and Treatment of Postmenopausal Osteoporosis2016. Endocr Pract 2016;22(4, Suppl 4):1-42

23 Eastell R, Rosen CJ, Black DM, Cheung AM, Murad MH, Shoback D. Pharmacological management of osteoporosis in postmenopausal women: an endocrine society clinical practice guideline. J Clin Endocrinol Metab 2019;104(05):1595-1622

24 Compston J, Cooper A, Cooper C, et al; National Osteoporosis Guideline Group (NOGG) UK clinical guideline for the prevention and treatment of osteoporosis. Arch Osteoporos 2017;12(01):43. Doi: $10.1007 / \mathrm{s} 11657-017-0324-5$

25 Sunyecz JA. The use of calcium and vitamin D in the management of osteoporosis. Ther Clin Risk Manag 2008;4(04):827-836

26 McClung MR. Bisphosphonates in osteoporosis: recent clinical experience. Expert Opin Pharmacother 2000;1(02):225-238

27 Dore RK. The RANKL pathway and denosumab. Rheum Dis Clin North Am 2011;37(03):433-452, vi-vii

28 Gennari L, Merlotti D, Nuti R. Selective estrogen receptor modulator (SERM) for the treatment of osteoporosis in postmenopausal women: focus on lasofoxifene. Clin Interv Aging 2010;5 (01):19-29

29 Miki RA, Oetgen ME, Kirk J, Insogna KL, Lindskog DM. Orthopaedic management improves the rate of early osteoporosis treatment after hip fracture. A randomized clinical trial. J Bone Joint Surg Am 2008;90(11):2346-2353

30 de Jong MR, Van der Elst M, Hartholt KA. Drug-related falls in older patients: implicated drugs, consequences, and possible prevention strategies. Ther Adv Drug Saf 2013;4(04):147-154

31 Panday K, Gona A, Humphrey MB. Medication-induced osteoporosis: screening and treatment strategies. Ther Adv Musculoskelet Dis 2014;6(05):185-202

32 Black DM, Cummings SR, Karpf DB, et al; Fracture Intervention Trial Research Group. Randomised trial of effect of alendronate on risk of fracture in women with existing vertebral fractures. Lancet 1996;348(9041):1535-1541

33 Cummings SR, Black DM, Thompson DE, et al. Effect of alendronate on risk of fracture in women with low bone density but without vertebral fractures: results from the Fracture Intervention Trial. JAMA 1998;280(24):2077-2082

34 Quandt SA, Thompson DE, Schneider DL, Nevitt MC, Black DMFracture Intervention Trial Research Group. Effect of 
e190 Hip Fractures in Malta Bugeja et al.

alendronate on vertebral fracture risk in women with bone mineral density T scores of- 1.6 to -2.5 at the femoral neck: the Fracture Intervention Trial. Mayo Clin Proc 2005;80(03):343-349

35 Jennings LA, Auerbach AD, Maselli J, Pekow PS, Lindenauer PK, Lee SJ. Missed opportunities for osteoporosis treatment in patients hospitalized for hip fracture. J Am Geriatr Soc 2010;58(04): 650-657

36 Cuschieri S, Grech S, Gatt R. Bisphosphonates: a cost benefit analysis patient. Malta Med J 2016;28(01):4-11
37 Huybrechts KF, Ishak KJ, Caro JJ. Assessment of compliance with osteoporosis treatment and its consequences in a managed care population. Bone 2006;38(06):922-928

38 Rabenda V, Vanoverloop J, Fabri V, et al. Low incidence of antiosteoporosis treatment after hip fracture. J Bone Joint Surg Am 2008;90(10):2142-2148

39 Lyles KW, Colón-Emeric CS, Magaziner JS, et al; HORIZON Recurrent Fracture Trial. Zoledronic acid and clinical fractures and mortality after hip fracture. N Engl J Med 2007;357(18):1799-1809 